\title{
Emotion
}

\section{When Seeing Is More Than Looking: Intentional Gaze Modulates Object Desirability}

Valeria Manera, Marco R. Elena, Andrew P. Bayliss, and Cristina Becchio

Online First Publication, April 21, 2014. http://dx.doi.org/10.1037/a0036258

CITATION

Manera, V., Elena, M. R., Bayliss, A. P., \& Becchio, C. (2014, April 21). When Seeing Is More Than Looking: Intentional Gaze Modulates Object Desirability. Emotion. Advance online publication. http://dx.doi.org/10.1037/a0036258 


\title{
When Seeing Is More Than Looking: Intentional Gaze Modulates Object Desirability
}

\author{
Valeria Manera \\ University of Nice-Sophia Antipolis and University of Turin
}

\author{
Marco R. Elena \\ University of Turin

\author{
Cristina Becchio \\ University of Turin and Istituto Italiano di Tecnologia
}

\author{
Andrew P. Bayliss \\ University of East Anglia
}

\begin{abstract}
Objects in the environment have a perceived value that can be changed through social influence. A subtle way to influence object evaluation is through eye gaze: Objects looked at by others are perceived as more likable than objects that are not looked at. In 3 experiments, we directly tested the hypothesis that this liking effect depends on the processing of the intentional relation between other's eye gaze and the object being looked at. To this end, we used a novel paradigm in which participants observed a face looking left or right behind an opaque barrier. Under all tested conditions, we found a gaze cueing effect on attention: Looked-at objects were categorized faster than looked-away objects. In contrast, observed gaze only led to a boost in affective evaluation for the target object when observers had the impression that the face could see the object behind the barrier, but not when observers had the impression that the face could not see the object. These findings indicate that observers make a sophisticated use of social gaze cues in the affective evaluation of objects: Objects looked at by others are liked more than objects looked away but only when others can see the objects.
\end{abstract}

Keywords: intentional gaze, object desirability, liking effect, gaze cueing

Humans use the reactions of others to help determine what is valuable: what to eat, what is dangerous, and what is attractive. Virtually all of the objects we interact with in our daily life, from food to money, have a perceived value, which can be changed through social influence (Campbell-Meiklejohn, Bach, Roepstorff, Dolan, \& Frith, 2010). A simple way to influence others' affective evaluations of objects is through eye gaze direction. Objects looked at by other people do not only receive more attention: They are also more likable (Bayliss, Paul, Cannon, \& Tipper, 2006).

Valeria Manera, CoBTek Laboratory, Faculty of Medicine, University of Nice-Sophia Antipolis and Center for Cognitive Science, Department of Psychology, University of Turin; Marco R. Elena, Center for Cognitive Science, Department of Psychology, University of Turin; Andrew P. Bayliss, School of Psychology, University of East Anglia; and Cristina Becchio, Center for Cognitive Science, Department of Psychology, University of Turin, and Department of Robotics, Brain, and Cognitive Sciences, Istituto Italiano di Tecnologia, Genova, Italy.

This work was supported by a grant from the Regione Piemonte, bando Scienze Umane e Sociali 2008, L.R. n.4/2006 to Cristina Becchio. Development of the MacBrain Face Stimulus Set was overseen by Nim Tottenham and supported by the John D. and Catherine T. MacArthur Foundation Research Network on Early Experience and Brain Development. Please contact Nim Tottenham at tott0006@tc.umn.edu for more information concerning the stimulus set.

Correspondence concerning this article should be addressed to Cristina Becchio, Department of Psychology, University of Turin, Via Po 14, 10123 Torino, Italy. E-mail: cristina.becchio@unito.it
In recent years there has been considerable progress in describing the features of this liking effect. For instance, objects looked at are preferred over those looked away from when the target face is smiling, but not when the face expresses disgust (Bayliss, Frischen, Fenske, \& Tipper, 2007; for a similar effect on face stimuli, see Jones, DeBruine, Little, Burriss, \& Feinberg, 2007). Similarly, liking is increased for objects looked by a trustworthylooking face. However, when the face appears untrustworthy, the effect is reversed: objects associated with an untrustworthy face are liked better when looked away from than when looked at (Treinen, Corneille, \& Luypaert, 2012; see also King, Rowe, \& Leonards, 2011). Object affective evaluation is also influenced by the pattern or history of gaze shifts. For example, liking is increased when the target face engages with the observer by making eye contact, then gazing at the object, and finally looking back at the observer (Van der Weiden, Veling, \& Aarts, 2010). Finally, liking effects can also be elicited during action observation, with objects that are seen to be manipulated being liked more than other objects - and such effects themselves can be dependent on social gaze (Hayes, Paul, Beuger \& Tipper, 2008; see also Lebreton, Kawa, Forgeot d'Arc, Daunizeau \& Pessiglione, 2012).

These findings suggest that social gaze-mediated liking is not purely the by-product of associative learning (see Bry, Treinen, Corneille, \& Yzerbyt, 2011), nor a mere consequence of observing averted gaze (Van der Weiden et al., 2010) or reflexive gaze cueing of attention (Bayliss et al., 2006). The extent to which the liking effect involves making assumptions about others' internal states remains, however, controversial (Corneille, Mauduit, Strick, \& Holland, 2009; see also Treinen et al., 2012). 
Other people's looking behavior can be represented on various different levels, ranging from the representation of its purely physical properties to the representation of seeing as an intentional act directed toward a specific object (Blakemore, Boyer, PachotClouard, Meltzoff, Segebarth, et al., 2003; Castelli, Happé, Frith, \& Frith, 2000; den Ouden, Frith, Frith, \& Blakemore, 2005; Pelphrey \& Morris, 2006). The aim of our present study was to seek direct evidence that the liking effect depends on the processing of the intentional relation between other's eye gaze and the object being looked at (Becchio, Bertone, \& Castiello, 2008).

A precondition for the intentional interpretation of gaze is that the target face not only looks in the direction of the object, but also can see the object (Nuku \& Bekkering, 2008; Teufel et al., 2009; Teufel, Alexis, Clayton, \& Davis, 2010). Is the social transmission of preference via gaze dependent on the belief that the other person can see the object? In other words, does liking require that the target face can see the object?

In the conventional gaze cueing paradigm used to study the gazeinduced liking effect the face directly looks at (and therefore can "see") the target object. It is therefore not possible to determine whether the effects on object evaluation depend on "seeing" the object or merely on "looking in the direction of" the object. To disentangle these two factors in the present study, we introduced a novel methodology based on an adaptation of the 'barriers paradigm' (Moll \& Tomasello, 2004). In the classic barriers paradigm, used to investigate whether and to what extent young infants understand looking as an act of seeing, the experimenter looks toward a target object that, from the infant's point of view, is hidden behind a barrier. If the infant follows the experimenter's gaze without understanding the intentional nature of looking, she will only look in the direction of the opaque barrier. However, if the infant understands that the adult is looking at something, she will move around the barrier to see what the adult is seeing. Critically, no response to the experimenter's head turn should be expected when the adult's line of sight is blocked in some way (e.g., with a blindfold or an opaque barrier) and the adult is therefore unable to see the object (Butler, Caron, \& Brooks, 2000).

Following this logic, in our task participants observed a face looking left or right at a location hidden from the participants' view by opaque occluders. Then the occluders moved over and obscured the face and an object either appeared at the location where the face had previously looked at (cued trials) or at the other side (uncued trials). The factor manipulated was the intentional contingency between gaze shift and object appearance; that is, the impression that the face could see or could not see the object behind the occluders. If the liking effect results from the processing of the intentional relation between others' eye gaze and the object being looked at, the ability of the face to see the object should be the critical variable in producing the effect. Therefore, irrespective of the presence of occluders, cued objects should be rated more favorably when observers have the impression that the face could see the object (intentional contingency preserved). By contrast, gaze shifts should have no influence on the affective appraisal of objects when observers have the impression that the face could not see the objects (intentional contingency disrupted).

We tested these predictions in three experiments. Experiment 1 was designed to replicate the liking effect for looked-at objects using the conventional gaze cueing paradigm in which the object appears while the face is looking one direction or the other with no barrier present at any time (i.e., object and face copresent; Bayliss et al., 2006). To test whether object liking depends on seeing rather than on mere looking in the direction of an object, in Experiment 2 and 3 we used barriers to manipulate the participants' impression of what the face could see during each trial (see Figure 1). In Experiment 2 the moving panels were used to prevent simultaneous visual access to the face and the object, while aiming to maintain the impression that the face could see the object. In Experiment 3, the panels moved in a slightly different way, aiming to give the impression that the face could not see the object at any time.

To briefly preempt our results, we found a gaze cueing effect on attention in each experiment, independently of the participants' impression that the face could or could not see the object. Moreover, we found a significant gaze liking effect in both Experiments 1 and 2, showing that the copresence of object and face is not necessary for the liking effect to emerge. Critically, however, the gaze liking effect was abolished in Experiment 3, meaning that the deciding factor for the emergence of the gaze liking effect is whether the face is able to see the object. Follow-up analyses confirmed that the subjective experience of the participant in these experiments is a key determinant in the emergence of the gaze liking effect. This finding provides a notable demonstration of the sophisticated use observers make of gaze cue in the affective evaluation of objects: Objects looked at by others are liked more than objects looked away but only when others can see the objects.

\section{Experiment 1}

Experiment 1 was designed to replicate the basic liking effect for objects being looked at (Bayliss et al., 2007, Experiment 1). This was needed to check whether the key manipulation of using occluding panels in Experiments 2 and 3 resulted in patterns of data-in terms of both attentional and affective consequences of observing other's gaze - comparable to those obtained using the original paradigm.

\section{Method}

Participants. Eighteen graduate and undergraduate students from the University of Turin ( 3 male and 15 female, mean age: 23.3 years, age range: $21-30$ years) volunteered for this study. All participants reported normal or corrected-to-normal vision, gave informed consent and were naïve with respect to the purpose of the study.

Apparatus and stimuli. Stimulus presentation and data collection were controlled by E-Prime2 (Psychology Software Tools, Inc. Sharpsburg, PA). Stimuli were presented $60 \mathrm{~cm}$ away from the participant on a 17.3-inch computer screen (16:9 aspect ratio, $1600 \times 900$ pixels resolution).

Twelve unfamiliar faces (six males, six females) with moderately positive facial expression were used as cue stimuli. The faces from the NimStim face set varied between 6.5 and $8.5 \mathrm{~cm}$ in width, and between 9.7 and $10.3 \mathrm{~cm}$ in height. They had three possible gaze directions: pupils straight ahead, pupils averted leftward, pupils averted rightward. The eye regions were approximately 0.5 $\mathrm{cm}$ high and $3.5 \mathrm{~cm}$ in width. The faces were presented in the center of the screen, and which face produced the cue on each trial was randomized. The fixation cross, which preceded each trial, measured $0.4 \times 0.4 \mathrm{~cm}$. 

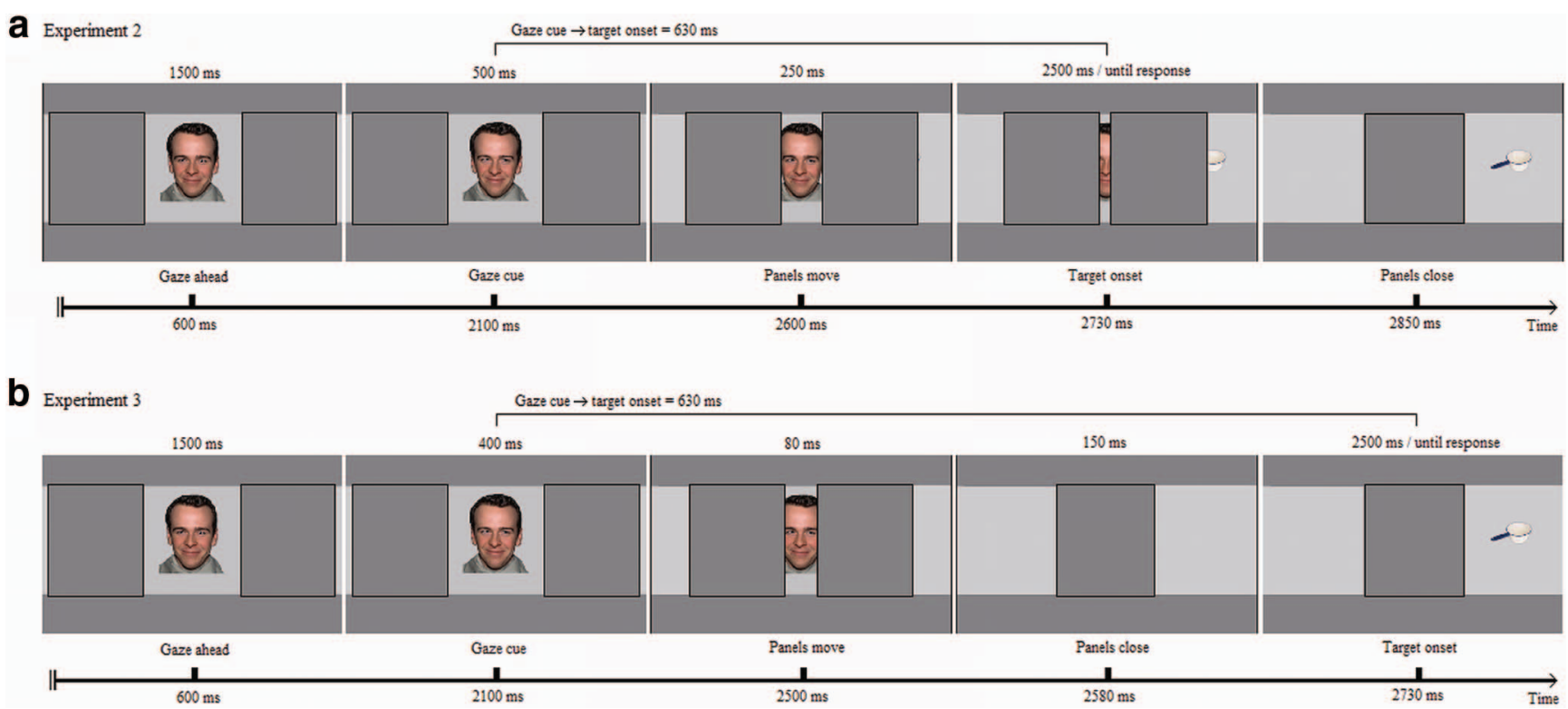

Figure 1. Illustration of the time course of an experimental trial (for a cued target) in Experiment 2 (a) and Experiment 3 (b). In both experiments, the trial started with a 600-ms fixation cross (not depicted), followed by the face with eyes gazing straight ahead for $1500 \mathrm{~ms}$, and by the gaze shift (a). In Experiment 2, $500 \mathrm{~ms}$ after the gaze shift the panels slid horizontally toward the center of the screen so as to cover the face stimulus. The object was uncovered $130 \mathrm{~ms}$ after the panel-sliding onset, that is, $630 \mathrm{~ms}$ after the gaze shift, when the eyes were completely hidden. The object remained visible until response or until $2500 \mathrm{~ms}$ elapsed (b). In Experiment 3, 400 ms after the gaze shift the panels slid horizontally toward the center of the screen, and covered the face completely after $80 \mathrm{~ms}$. The target appeared instantaneously $150 \mathrm{~ms}$ after the closure of the panels, that is, 630 $\mathrm{ms}$ after the gaze shift, and remained visible until response or until $2500 \mathrm{~ms}$ elapsed.

The target stimuli consisted of 28 images of household objects (Bayliss et al., 2006, 2007): 14 garage items (e.g., a saw, a screwdriver, a drill), and 14 kitchen items (e.g., a saucepan, a spoon, a mug). Images were digitally manipulated in Adobe Photoshop to create four different color versions of each object (blue, red, yellow, and green). Thus, there were a total of 112 possible target stimuli that could appear in their original orientation or flipped about the vertical axis. Objects varied between $1.5 \mathrm{~cm}$ and $5.5 \mathrm{~cm}$ in width, and between $1.0 \mathrm{~cm}$ and $3.9 \mathrm{~cm}$ in height. They were presented on the left or on the right of the cueing face, so that the center of the target was in line with gaze direction and approximately $12.5 \mathrm{~cm}$ from the center of the screen.

For the recording of preference ratings of the stimuli, a chart (in Italian) was presented, with the question "How much did you like that object?" at the top of the screen and with a column of numbers from 9 to 1 , headed by the words "Like very much" at the top and the words "Don't like at all" at the bottom of the screen.

Design. We used a within-subjects design with cueing as the within-subject factor. The cueing factor was whether the target object was looked at (cued), or looked away from (uncued). The manner in which the targets (kitchen/garage items) were presented to each participant was controlled as in previous reports (Bayliss et al., 2007). Although each object could appear in four colors, each participant would see only two color versions of each object: One version of the object would be looked at by the face stimulus each time it appeared, whereas the other version would be looked away from each time it appeared. To compensate for any problems for which the participants might simply rate objects in their favorite color more highly, we randomized which color was selected for each target, for each participant. Furthermore, which exemplar of each colored target would serve as the consistently cued item and the consistently uncued item across six exposures was also randomly assigned.

Procedure. Participants completed six blocks, five standard cuing blocks followed by a final rating block. In the standard cueing trials of Blocks 1 to 5, the participants were asked to fixate the fixation cross, to refrain from eye movements, to ignore the uninformative gaze cue, and to respond to the target as quickly as possible. Each trial started with a $600-\mathrm{ms}$ fixation cross. The face would then appear, with eyes gazing straight ahead for $1500 \mathrm{~ms}$. Then, $500 \mathrm{~ms}$ before target onset, the eyes would move to the left or the right. The target remained visible until response or until $2500 \mathrm{~ms}$ had elapsed. Participants used the "h" and "spacebar" keys to respond to the category of the target object. Whether " $h$ " corresponded to "Garage" or "Kitchen" items was counterbalanced between participants. At response, a tone was sounded to give feedback on performance (a "bell" for correct and a "buzzer" for incorrect/timeout). Finally, a 500-ms blank interval preceded the next trial. After 10 practice trials (using different target objects) participants completed five blocks of 56 trials, where each target was viewed once in each block.

In the final (sixth) rating block, the procedure changed slightly. The participants were now informed that following their target categorization response, a blank screen would be presented $(500 \mathrm{~ms})$, followed by a ratings screen. They were required to rate the item they had just responded to, so that higher scores were given to objects that 
they preferred. They were told to select a number from 1 to 9 , and to press the corresponding key on the keyboard. After their liking rating, another blank screen $(500 \mathrm{~ms})$ preceded the next trial.

In total, therefore, the participants completed 336 trials of the gaze-cueing procedure, being exposed six times to 28 cued targets and six times to 28 uncued targets. They rated each of these 56 targets once in the final (sixth) block.

\section{Results and Discussion}

Gaze cueing of attention. Accuracy in categorizing the targets over the six blocks was $97.3 \%$ (all participants reached our criterion of $90 \%$ accuracy; ranging from $92.0 \%$ to $100 \%$ of correct responses). For errors, the effect of cueing was nonsignificant, $t_{(17)}=-.13, p=$ $.898,{ }^{1} d_{z}=.03$. Analysis of mean reaction times (RTs) revealed the standard gaze cueing effect, with RTs being shorter to cued targets $(M=717 \mathrm{~ms}, S D=104 \mathrm{~ms})$ than to uncued targets $(M=767 \mathrm{~ms}$, $S D=102 \mathrm{~ms}$ ), $t_{(17)}=-9.63, p<.001, d_{z}=2.27$ (see Figure 2). Hence, we replicated the standard gaze cueing effect (Driver et al., 1999; Friesen \& Kingstone, 1998; see Frischen et al., 2007, for review).

Object ratings. Ratings given in the sixth block were compared for cued and uncued items. In line with previous studies (Bayliss et al., 2006, 2007), we found a significant effect of cueing on object ratings, $t_{(17)}=2.28, p=.036, d_{z}=.54$, with the items that were consistently looked at throughout the experiment being rated more preferable $(M=5.51, S D=.82)$ than were the items consistently looked away from $(M=5.29, S D=.86$; see Figure 2$)$.

\section{Experiment 2}

Experiment 1 confirmed that objects that are consistently looked at by other persons are rated as more likable compared to objects consistently not looked at, replicating previous work (Bayliss et al., 2006, 2007). A critical test for the hypothesis that liking depends on seeing rather than on mere looking is to examine whether, in the absence of directly observing the face gaze toward the object, seen objects are nevertheless rated as more likable compared with unattended objects. To this aim, in Experiment 2 we introduced horizontal occluding panels. At the beginning of the trial, the occluding panels were presented on the left and the right of the cueing face, so that the face was fully visible in between. After the gaze shift occurred, the panels moved horizontally toward the center of the screen so as to cover the face and to reveal the object on the left or right side. As a result, the eyes and the object were never simultaneously visually available to the observer. However, because the object emerged from behind the occluding panels, we expected that participants would still have the impression that, on cued trials, the face had been able to see the object (see Figure 1). We predicted that-despite the drastic manipulation produced by the introduction of the moving panelsobserved gaze direction would still have an impact on spatial attention, facilitating RTs to cued objects relative to uncued objects (Driver et al., 1999; Friesen \& Kingstone, 1998). Moreover, if seeing is critical for the liking effect, participants should still rate more favorable objects looked at than objects looked away even if the gaze behavior toward the object can only be inferred.
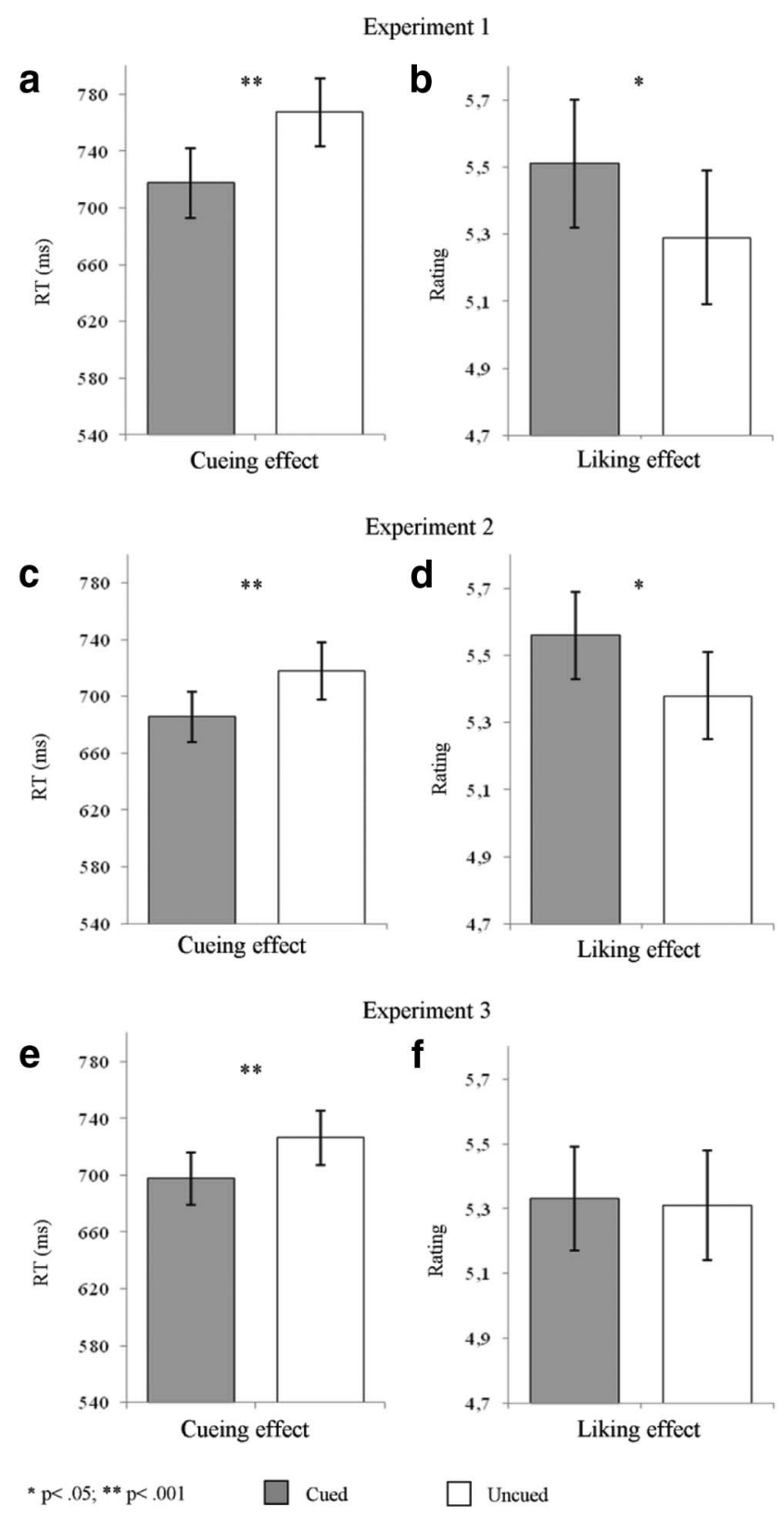

Figure 2. a, c, e: Bar charts (with standard error bars) illustrating the average reaction times (RTs) for cued (gray) and uncued (white) items in the three experiments (b, d, f). Bar charts (with standard error bars) illustrating the average ratings (between 1 and 9) for cued (gray) and uncued (white) items in the three experiments.

\section{Method}

Participants. Forty-nine graduate and undergraduate students from the University of Turin volunteered for this study. Although we anticipated that our procedure would give the impression that the face could see the object on cued trials, our postexperiment manipulation check-whereby we asked "Could the face see the

\footnotetext{
${ }^{1} p$ values always refer to two-tailed tests.
} 
objects when the eyes looked toward the same side of the screen at which the object appeared?"-found that 21 participants were convinced that the face could not see the objects on cued trials. ${ }^{2}$ These participants were thus excluded from the current analysis. One additional participant exceeded $10 \%$ of errors in the categorization task and was also excluded. The final sample therefore consisted of 27 participants (four male and 23 female, mean age $=$ 20.2 years, age range $=19-28$ years). All participants reported normal or corrected-to-normal vision, gave informed consent, and were naïve with respect to the purpose of the study. None of the Experiment 2 participants had participated in Experiment 1.

Stimuli and procedure. The stimuli, design, and procedure were identical to Experiment 1, except that two horizontal occluding panels (measuring $11.7 \times 13.0 \mathrm{~cm}$ ) were added. At the beginning of the trial, the occluding panels were presented on the left and the right of the cueing face, so that the face was fully visible in between. Five hundred milliseconds after the gaze shift, the panels slid horizontally toward the center of the screen so as to cover the face stimulus and to reveal an object on the left or right side. The target was uncovered $130 \mathrm{~ms}$ after the panel-sliding onset (i.e., $630 \mathrm{~ms}$ after the gaze shift), when the eyes were completely hidden by the barriers (see Figure 1). As a result, the eyes and the object were never visually available at the same time. However, because the object emerged from behind the occluding panels on the same plane of the face, participants still had the impression that, on cued trials, the face had been able to see the object (see Figure 1). The target remained visible until response or until 2500 ms elapsed. After completing the experimental session, participants were asked whether during the experiment they had had the impression that the face could see the object on cued trials. Only participants reporting that the face could see the object in cued trials were included in the analysis (see Participants section).

\section{Results and Discussion}

Gaze cueing. Accuracy in categorizing the targets over the six blocks was $96.6 \%$ (ranging from $91.1 \%$ to $100 \%$ of correct responses). For errors, the effect of cueing was nonsignificant, $t_{(26)}=-.59, p=.558, d_{z}=.11$. Analysis of RTs revealed the standard gaze-cueing effect, with RTs being shorter to cued targets $(M=686 \mathrm{~ms}, S D=93 \mathrm{~ms})$ than to uncued targets $(M=718 \mathrm{~ms}$, $S D=104 \mathrm{~ms}$ ), $t_{(26)}=-5.54, p<.001, d_{z}=1.07$ (see Figure 2).

Object ratings. Consistent with Experiment 1, there was a significant effect of cueing on object ratings, $t_{(26)}=2.62, p=$ $.015, d_{z}=.50$, with the items that were consistently looked at throughout the experiment being rated more preferable $(M=5.56$, $S D=.65)$ than were the items consistently looked away from $(M=5.38, S D=.67)$. This demonstrates that the copresence of gaze and object is not necessary for the liking effect to occur-at least in individuals who report that they had the subjective impression that the face could see the cued objects.

Hence, despite the introduction of horizontal moving panels that prevented simultaneous visual access to the face and the object, Experiment 2 replicated both the standard effect of gaze cues on attention and on affective evaluations.

\section{Experiment 3}

Provided that observers know that the object has been seen by the face stimulus, the liking effect is observed even when, during gaze shift, the critical object is hidden from view (Experiment 2). To investigate whether seeing is indeed the key factor in determining the effect, in Experiment 3 we manipulated the timing of the appearance of the object after the gaze shift so that instead of emerging from behind the occluding panels, the object instantaneously appeared "from nothing" after the closure of the panels on the face stimulus. Under these circumstances, the face stimulus is supposed to be unable to see the object behind the panels. We therefore predict that despite moving panels were identical to those of Experiment 2, no liking effect should be observed in Experiment 3 . However, as the gaze direction can be correctly processed by the visual attention system, a gaze cueing effect on attention should nevertheless be observed.

\section{Method}

Participants. Thirty-four graduate and undergraduate students from the University of Turin volunteered for this study. Although we anticipated that our procedure would give the impression that the face could not see the object in the cued trials, our postexperiment manipulation check whereby we asked "Could the face see the objects when the eyes looked toward the same side of the screen at which the object appeared?" revealed that seven participants were convinced that the face could see the objects on cued trials and were therefore excluded from the present analysis. One additional participant was excluded because more than $10 \%$ of her judgments in the categorization task were incorrect. The final sample comprised 26 participants (six male and 20 female, mean age $=24$ years, age range $=19-38$ years). All participants reported normal or corrected-to-normal vision, gave informed consent and were naïve with respect to the purpose of the study. None of the Experiment 3 participants had participated in Experiments 1 and 2 .

Stimuli and procedure. The stimuli, the design, and the procedure were identical to Experiment 2, except for the timing (onset and speed) of the panels' sliding. At the beginning of each trial, the occluding panels were presented on the left and the right of the cueing face as in Experiment 2. The barrier sliding was faster compared to Experiment 2, and the target appeared instantaneously $150 \mathrm{~ms}$ after the closure of the occluding panels on the face stimulus (see Figure 1). This manipulation generated the impression that the object appeared suddenly from nothing after the panel closure, instead of being gradually revealed by the panels' sliding. To keep the time that elapsed between gaze shift and object onset constant as compared to Experiment $2(630 \mathrm{~ms})$, the panels' sliding started $400 \mathrm{~ms}$ after the gaze shift and took $80 \mathrm{~ms}$ to complete. As in Experiment 2, the face stimulus and object were never visually available at the same time. The objects remained visible until response or until $2500 \mathrm{~ms}$ elapsed. After completing the experimental session, participants were asked whether during the experiment they had had the impression that the face could see the

\footnotetext{
${ }^{2}$ As detailed below, in Experiment 2, the eyes and the object were never simultaneously visually available to the observer. It is thus possible that some participants did not form the impression that the face could see the object or only formed a very weak impression. The manipulation check ensured that only participants reporting the impression that the face could see the object in cued trials were included in the analysis of Experiment 2. For an alternative way to interrogate the data according to the impression reported by participants, please see the Comparing Performance Across Experiments section.
} 
object on cued trials. Only participants reporting that the face could not see the object in cued trials were included in the analysis.

\section{Results and Discussion}

Gaze cueing. Accuracy in categorizing the targets over the six blocks was $96.6 \%$ (ranging from $93.8 \%$ to $99.4 \%$ of correct responses). For errors, the effect of cueing was nonsignificant, $t_{(25)}=-.70, p=.488, d_{z}=.14$. Analysis of RTs revealed the standard gaze cueing effect, with RTs being shorter to cued items ( $M=698 \mathrm{~ms}, S D=95 \mathrm{~ms})$ than to uncued items $(M=726 \mathrm{~ms}$, $S D=98 \mathrm{~ms}$ ), $t_{(25)}=-4.34, p<.001, d_{z}=.85$ (see Figure 2 ). This means that although these participants did not think that the face could see the cued objects, they nevertheless processed the gaze cue and shifted spatial attention in the direction of the cue.

Object ratings. The effect of cueing on object ratings was not statistically significant, $t_{(25)}=.22, p=.832, d_{z}=.04$, with the cued items $(M=5.33, S D=.83)$ liked as much as the uncued items $(M=$ $5.31, S D=.85$; see Figure 2). These findings suggest that when the face is unable to see the object, gaze shifts do not evoke liking effects. By contrast, a strong gaze cueing effect emerges. This is an important aspect of our data, as it rules out the possibility that the absence of liking might be due to the disrupted encoding of gaze direction per se: If occluding panels interfered with the encoding of gaze direction, no gaze cueing should emerge.

\section{Comparing Performance Across Experiments}

Each of our experiments showed that gaze cueing of attention was robust despite our occluder manipulations. Critically, although gaze-cueing of attention was significantly stronger in Experiment 1 than in Experiment 2 (mean difference in RTs between cued and uncued items: $50 \mathrm{~ms}$ vs. $\left.32 \mathrm{~ms} ; t_{(43)}=-2.12, p=.040\right)$ and in Experiment 3 (50 ms vs. $\left.29 \mathrm{~ms} ; t_{(42)}=-2.34, p=.024\right)$, no significant difference was found between Experiments 2 and 3 (32 ms vs. $\left.29 \mathrm{~ms} ; t_{(51)}=-0.40, p=.688\right)$. This indicates that overall, the presence of barriers reduced gaze cueing somewhat, but the method of the target's appearance from behind the barriers did not modulate the effect of gaze cueing on attention.

In line with a priori predictions, the effect of gaze cueing on object ratings was weaker in Experiment 3 than in Experiment 1 $\left(.02\right.$ vs. $\left..22 ; t_{(42)}=-1.81, p=.077\right)$ and in Experiment 2 (.02 vs. $\left..18 ; t_{(51)}=-1.72, p=.092\right)$; both results were trending toward significance. On the contrary, no significant difference in object rating was found between Experiment 2 and 1 (.18 vs. .22 on a 9 -point scale; $\left.t_{(43)}=.37, p=.716\right)$. These results suggest that the effect of gaze shift on object liking does depend on the impression the face can see the object. However, we were concerned that the exclusion of participants who did not have the subjective impression that the face could see $(N=21$; Experiment 2$)$ or could not see $(N=7$; Experiment 3$)$ the cued target objects might have a bearing on our findings. Moreover, although the liking effect was numerically weaker in Experiment 3 than in both Experiments 1 and 2, this cross-experiment comparison did not reach statistical significance. We therefore conducted further analyses that take into account these two points to seek supplementary, reinforcing support for our hypotheses. ${ }^{3}$

If the liking effect is critically dependent on the seeing impression, then it should emerge when participants judge that the face could see the object, but not when they report the impression that the face stimulus was unable to see the object. An alternative approach to interrogate the data of Experiments 2 and 3 is therefore to analyze the data according to the judgments made by participants, irrespective of the particular barrier paradigm used. In Experiment 2 the object emerged from behind occluding panels, to give the impression that, on cued trials, the face was able to see the object. Twenty-one participants reported, however, the impression that the face was unable to see the object. Similarly, in Experiment 3, seven participants reported the face could see the object, despite the fact that the object appeared "from nothing" after the closure of the panels.

To test the influence of subjective report, in a second analysis, we compared gaze cueing and object ratings in participants who believed the face could see the object from Experiments 2 and $3(N=33$, six males and 27 females, mean age: 21 years) and participants from Experiment 2 and 3 who believed the face could not see the object ( $N=46$, 11 males and 35 females, mean age: 22 years), with the grouping factor being subjective impression (see Figure 3 ). Two additional participants (one for Experiment 2, one for Experiment 3) were excluded from the analysis because more than $10 \%$ of their judgments in the categorization task were incorrect.

\section{Results}

Gaze cueing. Analysis of variance (ANOVA) on RTs with cueing (cued vs. uncued) as within-subject factor and subjective impression (face can see the object vs. face cannot see the object) as between-subjects factor revealed a significant main effect of cueing, $F_{(1,77)}=77.13, p<.001$, with RTs being shorter to cued items than to uncued items. Importantly, no significant interaction between cueing and subjective impression was found, $F_{(1,77)}=$ $.49, p=.485$, thus suggesting that the gaze-cueing effect was not affected by the subjective impression that the face could or could not see the object. Accordingly, paired-sample contrasts showed the standard gaze cueing effect both for participants who believed that the face could see the object (33 ms cueing effect on RTs, $\left.t_{(32)}=6.18, p<.001, d_{z}=1.07\right)$ and for participants who believed that the face could not see the object (29 ms cueing effect on RTs, $\left.t_{(45)}=6.28, p<.001, d_{z}=.93\right)$.

Object ratings. ANOVA on object ratings with cueing (cued vs. uncued) as within-subject factor and subjective impression (face can see the object vs. face cannot see the object) as betweensubjects factor revealed a main effect of cueing trending toward significance, $F_{(1,77)}=3.35, p=.071$. However, this was moderated by a highly significant interaction effect between cueing and subjective impression, $F_{(1,77)}=9.91, p=.002$. To further explore the interaction effect, we performed paired-sample contrasts on object ratings for cued versus uncued items, separately for participants who had the subjective impression that the face could see the object and for participants who had the subjective impression that the face could see the object. Consistent with our hypothesis, participants who, irrespective of the paradigm used, reported the impression that the face could see the object, showed a highly

\footnotetext{
${ }^{3}$ An alternative way of examining the hypothesis would have been to manipulate the critical factor (i.e., impression that the face can see the object) within subjects. With a within-subjects design, however, the effect of the seeing condition may have easily carried over to the nonseeing condition, limiting the interpretation of the results.
} 

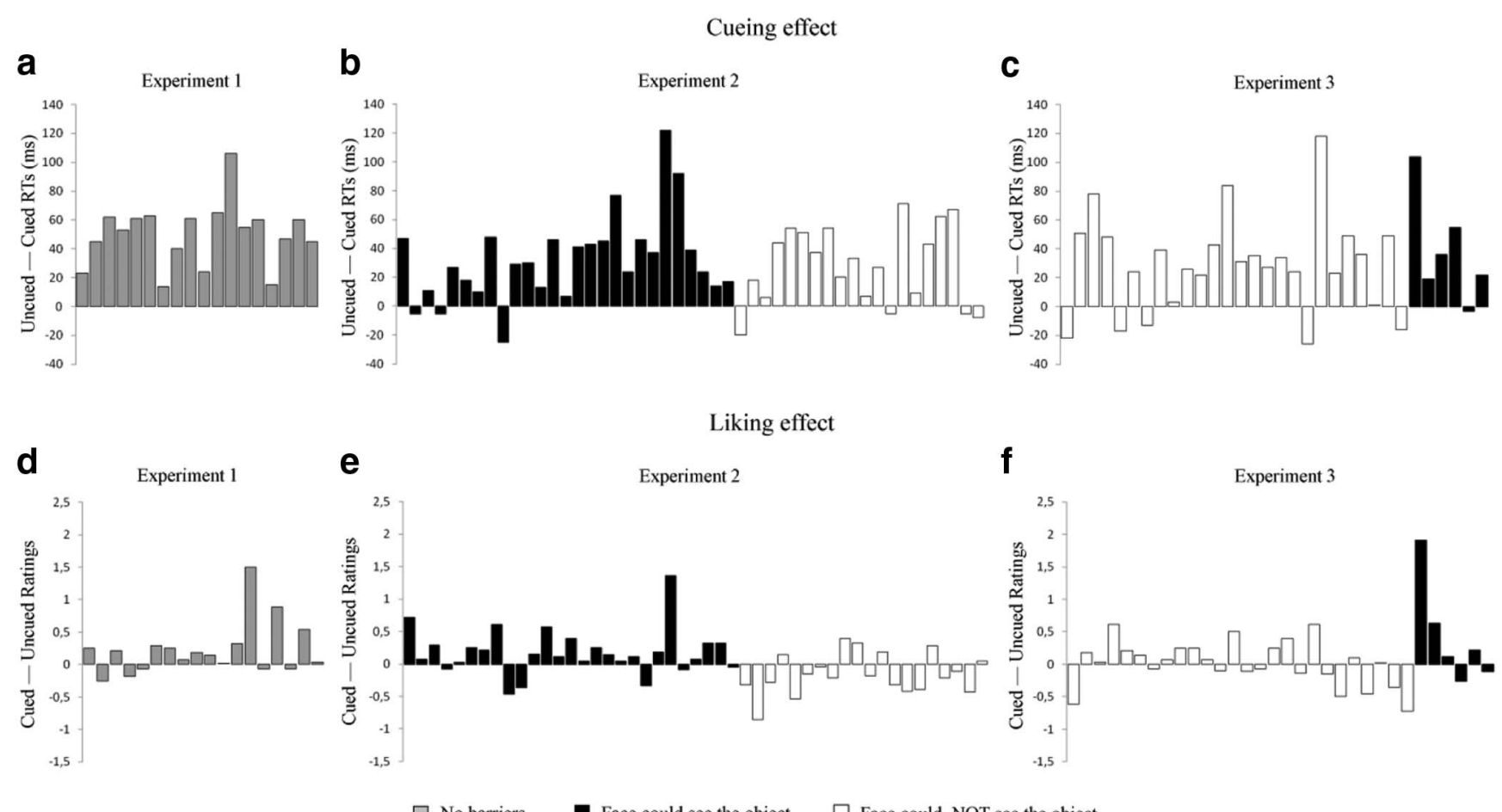

\begin{abstract}
$\square$ No barriers
Face could see the object $\quad \square$ Face could NOT see the object

Figure 3. a, b, c: Bar charts illustrating the gaze cueing of attention shown by individual participants. Bars above the $\mathrm{x}$-axis illustrate that RTs were shorter to cued items than to uncued items (d, e, f). Bar charts illustrating the effect of cueing on object ratings shown by individual participants. Bars above the x-axis illustrate that a participant rated cued items higher than uncued items. Bars are ordered by subjective impression and then by order of recruitment.
\end{abstract}

significant liking effect, with cued items $(M=5.53, S D=.88)$ rated higher than uncued items $(M=5.30, S D=.90), t_{(32)}=2.80$, $p=.009, d_{z}=.49$. On the contrary, participants who believed that the face could not see the object liked the cued items $(M=5.13$, $S D=.83)$ as much as the uncued items $(M=5.19, S D=.82)$, $t_{(45)}=-1.18, p=.244, d_{z}=.18$.

\section{General Discussion}

When we see someone change their direction of gaze, we spontaneously follow their eyes (Driver et al., 1999; Friesen \& Kingstone, 1998). Similar cueing effects are evoked by nongaze cues, such as arrows (Bayliss, di Pellegrino, \& Tipper, 2005; Bayliss \& Tipper, 2005; Bayliss, Bartlett, Naughtin, \& Kritikos, 2011; Frischen et al., 2007). Yet, there is an important effect produced by gaze cues which arrows cues fail to produce: Stimuli perceived to be looked at by others acquire a positive valence (Bayliss et al., 2006). A so far little-explored question is whether or not this liking effect requires that the looking behavior of others is conceptualized as an intentional act of seeing.

A limitation inherent in the conventional gaze cueing paradigm is that, because the object appears while the face is looking in one direction or the other, it not possible to determine whether liking depends on looking in the direction of or on seeing the object. Evidence for modulation by emotional expression (e.g., Bayliss et al., 2007) and face trustworthiness (Treinen et al., 2012) is compatible with the intentional processing of other's gaze. However, because in these studies the target face directly looked at the object, a mechanism that merely links the coincidence of emotional expression and looking direction to the object cannot be excluded. Similarly, without assuming any attribution of seeing, it could be hypothesized that direct gaze to the observer increases the perceived desirability of the gazed-at object (Van der Weiden, et al., 2010) because it enhances the saliency of the subsequent gaze shift.

To directly test the hypothesis that liking effect requires that the target face sees the object (in contrast to merely looking in the direction of the object), in three experiments we systematically manipulated the intentional contingency between gaze shift and object appearance. Experiment 1 replicated the liking effect for looked-at objects using the conventional gaze cueing paradigm. In Experiments 2 and 3, we used a modified barriers paradigm (Moll \& Tomasello, 2004) to prevent simultaneous visual access to the eyes and the object and alter the impression that face could see the object. We found that in the presence of occluding panels, gaze shifts influenced object desirability, but only when the intentional contingency between gaze shift and object appearance was preserved and participants had the impression that the face stimulus could see the object behind the panel. When the intentional contingency between gaze shift and object appearance was disrupted and participants had the impression that the face stimulus was unable to see the object, no liking effect was observed. 


\section{Seeing Is More Than Looking}

Previous studies provide indication that high-level social cognition such as attributing a seeing mental state can influence the allocation of spatial attention in response to a gaze cue (Nuku \& Bekkering, 2008; Ristic \& Kingstone, 2005; Teufel et al., 2010). For instance, reflexive gaze following has been found to be stronger when observers believe that the face stimulus wears transparent goggles (and thus can see) than when they believe that the goggles are opaque and the face can therefore not see (Teufel et al., 2010). As transparent and opaque goggles looked identical from the perspective of an onlooker, this indicates that coding of gaze during alignment of attention is differently influenced by the belief that the face can or cannot see. In the present study, we manipulated the impression that the face stimulus could or could not see the object and found that that attribution of seeing is critical for gaze shifts to influence the affective evaluation of objects. Object liking requires that not only the face looks in the direction of the object but can see the object. In contrast, gaze cueing was revealed independently from seeing, that is, both when the face stimulus could see the object (Experiment 2) and when the face could not see the object (Experiment 3).

At first glance, the finding that gaze cueing emerged independently from seeing may seem to be at odds with the results of Teufel and colleagues (2010), who suggested that attribution of seeing does influence reflexive gaze following. This seeming contradiction can be resolved upon consideration of differences in the experimental designs. Teufel and colleagues manipulated beliefs about another person's ability to see the entire visual field (i.e., perceive). In contrast, we manipulated the impression that the face could or could not see a particular element of the environment-the target object, that is, the interpretation of seeing as object-directed. In both Experiments 2 and 3 the face stimulus had the visual ability to see, however-critically-only in Experiment 2, but not in Experiment 3, the face was able to see the target object.

Processing of object-directedness is crucial for linking the perception of the gaze shift with a theory about the gaze shift's significance in terms of the other person's intentions (Pelphrey \& Morris, 2006). Activity within the superior temporal sulcus-a key region in social perception-is sensitive to object-directedness of gaze shift (Pelphrey, Singerman, Allison, \& McCarthy, 2003) and there is evidence that object-directed gaze is used to infer motor intentions (Pierno, Becchio, Wall, Smith, Turella et al., 2006). Together with previous result, our findings suggest that attribution of intentionality modulates affective responses to another's person gazing behavior: Objects looked at by others are liked more than objects looked away but only when the gazing behavior of others is interpreted as an intentional act.

\section{Copresence of Eyes and Object}

The time elapsed between gaze shift and object appearance being equal (630 ms), object liking was enhanced when observers believed that the face stimulus could see the object (Experiment 2), but not when they believed that the face could not see the object (Experiment 3). An interesting implication of these results is that, provided the face stimulus can see the object, copresence between gaze and object is not necessary for the liking effect to emerge. In Experiment 2, moving occluders prevented simultaneous access to the eyes and the object, yet cued objects were rated more favorably compared to uncued objects. This finding suggests that others' gaze behavior may influence the desirability of objects not visible (from the observer's point of view) when the gaze shift takes place.

Increased liking for objects looked at by others has been proposed to reflect an advantageous social adaptation: Others look toward objects because they like them (Baron-Cohen, Campbell, KarmiloffSmith, Grant, \& Walker, 1995); if they look away from them, they probably do not. It would seem adaptive that observers accommodate others' preferences in their own behavior as it aids in assessing information about the environment and objects within it (Emery, 2000). The finding that copresence of gaze and object is not necessary for the liking effect to occur as long as the face is believed to be able to see the object reinforces this idea and suggests that the looking behavior of others may contribute to establish preferences for objects that are not immediately accessible to the gaze of the observer. In this way, gaze-mediated liking may not only alter the value of specific visible objects but also predetermine a preference for objects temporarily occluded from view.

But when can others' preferences be trusted? Face trustworthiness has been shown to play a moderating role in establishing object preferences (King et al., 2011; Treinen et al., 2012). At a more basic level, however, a critical condition for trusting the other's preference is that he or she can see the object. When the face is not able to see the object, no inference about the other's preferences is possible and no influence on affective appraisal of objects should therefore be expected. In line with this, results of Experiment 3 indicate that liking was abolished when observers believed that the face could not see the object.

The influence of gaze shifts on object evaluation has been likened to a form of mimetic desire (Corneille et al., 2009): People's desires are not autonomous but rather based on the imitation of what they perceive other people desire. Our results suggest that this mimesis might extend over time and, at least as far as object preference is concerned, may lead to imitate others' preferences even before the object of their preference is known.

\section{Conclusions}

Gaze provides information regarding both the outside and the inside. From the gaze of another person we can infer information about relevant events and objects within the external environment. At the same time, from observing another person's gaze we are able to access information related to intentions and mental states of the other person. For instance, we can infer what another person might be interested in and what she might desire (e.g., Lee, Eskritt, Symons, \& Muir, 1998). The liking effect has been proposed to arise from the interplay of these two aspects: We tend to transfer onto objects the attitude we read into the eyes of others (Becchio et al., 2008). Our findings support this assumption demonstrating that whereas gaze cueing depends on mere looking, liking results from the processing of the intentional relation between others' eye gaze and the object being looked at. Consistent with earlier reasoning on temporal dynamics of gaze perception (Itier \& Batty, 2009), it is tempting to speculate that gaze-cueing and object liking reflect successive steps of gaze processing. Provided that the face is able to see (Teufel et al., 2010), gaze cueing emerges as a fast and almost automatic response to gaze shift during early steps of gaze processing. Object liking relates to a more cognitive step that influences emotional responses to the object, is 
context sensitive, and only occurs when observers have the impression that the face can see the object.

\section{References}

Baron-Cohen, S., Campbell, R., Karmiloff-Smith, A., Grant, J., \& Walker, J. (1995). Are children with autism blind to the mentalistic significance of the eyes? British Journal of Developmental Psychology, 13, 379-398. doi:10.1111/j.2044-835X.1995.tb00687.x

Bayliss, A. P., Bartlett, J. Naughtin, C. K., \& Kritikos, A. (2011). A direct link between gaze perception and social attention. Journal of Experimental Psychology: Human Perception and Performance, 37, 634-644. doi:10.1037/a0020559

Bayliss, A. P., di Pellegrino, G., \& Tipper, S. P. (2005). Sex differences in eye gaze and symbolic cueing of attention. The Quarterly Journal of Experimental Psychology, 58A, 631-650. doi:10.1080/02724980443000124

Bayliss, A. P., Frischen, A., Fenske, M. J., \& Tipper, S. P. (2007). Affective evaluations of objects are influenced by observed gaze direction and emotional expression. Cognition, 104, 644-653. doi:10.1016/ j.cognition.2006.07.012

Bayliss, A. P., Paul, M. A., Cannon, P. R., \& Tipper, S. P. (2006). Gaze cueing and affective judgments of objects: I like what you look at. Psychonomic Bulletin \& Review, 13, 1061-1066. doi:10.3758/ BF03213926

Bayliss, A. P., \& Tipper, S. P. (2005). Gaze and arrow cueing of attention reveals individual differences along the autism-spectrum as a function of target context. British Journal of Psychology, 96, 95-114. doi:10.1348/ $000712604 X 15626$

Becchio, C., Bertone, C., \& Castiello, U. (2008). How the gaze of others influences object processing. Trends in Cognitive Sciences, 12, 254258. doi:10.1016/j.tics.2008.04.005

Blakemore, S. J., Boyer, P., Pachot-Clouard, M., Meltzoff, A., Segebarth, C., \& Decety, J. (2003). The detection of contingency and animacy from simple animations in the human brain. Cerebral Cortex, 13, 837-844. doi:10.1093/cercor/13.8.837

Bry, C., Treinen, E., Corneille, O., \& Yzerbyt, V. (2011). Eye'm lovin' it! The role of gazing awareness in mimetic desires. Journal of Experimental Social Psychology, 47, 987-993. doi:10.1016/j.jesp.2011.03.023

Butler, S. C., Caron, A. J., \& Brooks, R. (2000). Infant understanding of the referential nature of looking. Journal of Cognition and Development, 1 , 359-377. doi:10.1207/S15327647JCD0104_01

Campbell-Meiklejohn, D. K., Bach, D. R., Roepstorff, A., Dolan, R. J., \& Frith, C. D. (2010). How the opinion of others affects our valuation of objects. Current Biology, 20, 1165-1170. doi:10.1016/j.cub.2010.04.055

Castelli, F., Happé, F., Frith, U., \& Frith, C. D. (2000). Movement and mind: A functional imaging study of perception and interpretation of complex intentional movement patterns. Neuroimage, 12, 314-325. doi:10.1006/nimg.2000.0612

Corneille, O., Mauduit, S., Strick, M., \& Holland, R. (2009). Liking products by the head of a dog: Perceived orientation of attention induces valence acquisition. Journal of Experimental Social Psychology, 45, 234-237. doi:10.1016/j.jesp.2008.07.004

den Ouden, H. E., Frith, U., Frith, C. D., \& Blakemore, S. J. (2005). Thinking about intentions. Neuroimage, 28, 787-796. doi:10.1016/j .neuroimage.2005.05.001

Driver, J., Davis, G., Ricciardelli, P., Kidd, P., Maxwell, E., \& BaronCohen, S. (1999). Gaze perception triggers reflexive visuospatial orienting. Visual Cognition, 6, 509-540. doi:10.1080/135062899394920

Emery, N. J. (2000). The eyes have it: The neuroethology, function and evolution of social gaze. Neuroscience and Biobehavioral Reviews, 24, 581-604. doi:10.1016/S0149-7634(00)00025-7
Friesen, C. K., \& Kingstone, A. (1998). The eyes have it! Reflexive orienting is triggered by nonpredictive gaze. Psychonomic Bulletin \& Review, 5, 490-495. doi:10.3758/BF03208827

Frischen, A., Bayliss, A. P., \& Tipper, S. P. (2007). Gaze-cueing of attention: Visual attention, social cognition and individual differences. Psychological Bulletin, 133, 694-724. doi:10.1037/0033-2909.133.4.694

Hayes, A. E., Paul, M. A., Beuger, B., \& Tipper, S. P. (2008). Self produced and observed actions influence emotion: The role of action fluency and eye gaze. Psychological Research, 72, 461-472. doi:10.1007/s00426-007-0125-3

Itier, R. J., \& Batty, M. (2009). Neural bases of eye and gaze processing: The core of social cognition. Neuroscience and Biobehavioral Reviews, 33, 843863. doi:10.1016/j.neubiorev.2009.02.004

Jones, B. C., DeBruine, L. M., Little, A. C., Burriss, R. P., \& Feinberg, D. R. (2007). Social transmission of face preferences among humans. Proceedings of the Royal Society B: Biological Sciences, 274, 899-903. doi:10.1098/rspb.2006.0205

King, D., Rowe, A., \& Leonards, U. (2011). I trust you; hence I like the things you look at: Gaze cueing and sender trustworthiness influence object evaluation. Social Cognition, 29, 476-485. doi:10.1521/soco.2011.29.4.476

Lebreton, M., Kawa, S., Forgeot d'Arc, B., Daunizeau, J., \& Pessiglione, M. (2012). Your goal is mine: Unravelling mimetic desires in the human brain. Journal of Neuroscience, 32, 7146-7157. doi:10.1523/ JNEUROSCI.4821-11.2012

Lee, K., Eskritt, M., Symons, L. A., \& Muir, D. (1998). Children's use of triadic eye gaze information for "mind reading." Developmental Psychology, 34, 525-539. doi:10.1037/0012-1649.34.3.525

Moll, H., \& Tomasello, M. (2004). Twelve- and 18-month-old infants follow gaze to spaces behind barriers. Developmental Science, 7, F1-F9. doi:10.1111/j.1467-7687.2004.00315.x

Nuku, P., \& Bekkering, H. (2008). Joint attention: Inferring what others perceive (and don't perceive). Consciousness and Cognition: An International Journal, 17, 339-349. doi:10.1016/j.concog.2007.06.014

Pelphrey, K. A., \& Morris, J. P. (2006). Brain mechanisms for interpreting the actions of others from biological-motion cues. Current Directions in Psychological Science, 15, 136-140. doi:10.1111/j.0963-7214.2006.00423.x

Pelphrey, K. A., Singerman, J. D., Allison, T., \& McCarthy, G. (2003). Brain activation evoked by perception of gaze shifts: The influence of context. Neuropsychologia, 41, 156-170. doi:10.1016/S00283932(02)00146-X

Pierno, A. C., Becchio, C., Wall, M. B., Smith, A. T., Turella, L., \& Castiello, U. (2006). When gaze turns into grasp. Journal of Cognitive Neuroscience, 18, 2130-2137. doi:10.1162/jocn.2006.18.12.2130

Ristic, J., \& Kingstone, A. (2005). Taking control of reflexive social attention. Cognition, 94, B55-B65. doi:10.1016/j.cognition.2004.04.005

Teufel, C., Alexis, D. M., Clayton, N. S., \& Davis, G. (2010). Mental-state attribution drives rapid, reflexive gaze following. Attention, Perception, \& Psychophysics, 72, 695-705. doi:10.3758/APP.72.3.695

Teufel, C., Alexis, D. M., Todd, H., Lawrance-Owen, A. J., Clayton, N. S., \& Davis, G. (2009). Social cognition modulates the sensory coding of observed gaze direction. Current Biology, 19, 1274-1277. doi:10.1016/ j.cub.2009.05.069

Treinen, E., Corneille, O., \& Luypaert, G. (2012). L-eye to me: The combined role of Need for Cognition and facial trustworthiness in mimetic desires Cognition, 122, 247-251. doi:10.1016/j.cognition.2011.10.006

Van der Weiden, A., Veling, H., \& Aarts, H. (2010). When observing gaze shifts of others enhances object desirability. Emotion, 10, 939-943. doi: $10.1037 / \mathrm{a} 0020501$

Received November 18, 2013

Revision received January 29, 2014

Accepted February 4, 2014 